\title{
Investigation of the roles of exosomes in colorectal cancer liver metastasis
}

\author{
$\mathrm{XIA} \mathrm{WANG}^{1}$, XIAOLING DING $^{1}$, LIJUAN NAN $^{1}$, YITING WANG $^{1}$, JING WANG $^{1}$, ZHIQIANG YAN $^{1}$, \\ WEI ZHANG ${ }^{1}$, JHHONG SUN ${ }^{3}$, WEI ZHU ${ }^{4}$, BING NI $^{5}$, SUZHEN DONG ${ }^{1,2}$ and LEI YU ${ }^{1}$ \\ ${ }^{1}$ Institute of Biomedical Engineering and Technology, Institutes for Advanced Interdisciplinary Research, \\ East China Normal University, Shanghai 200062; ${ }^{2}$ Shanghai Engineering Research Center of Molecular Therapeutics \\ and New Drug Development, Institutes for Advanced Interdisciplinary Research, East China Normal University, \\ Shanghai 200062; ${ }^{3}$ Sir Run Run Shaw Hospital, School of Medicine, Zhejiang University, Hangzhou 310016; \\ ${ }^{4}$ Department of General Surgery, Zhongshan Hospital, Fudan University, Shanghai 200032; \\ ${ }^{5}$ School of Life Science, East China Normal University, Shanghai 200241, P.R. China
}

Received January 7, 2015; Accepted February 19, 2015

DOI: $10.3892 /$ or.2015.3843

\begin{abstract}
The leading cause of death among cancer patients is tumor metastasis. Tumor-derived exosomes are emerging as mediators of metastasis. In the present study, we demonstrated that exosomes play a pivotal role in the metastatic progression of colorectal cancer. First, a nude mouse model of colorectal cancer liver metastasis was established and characterized. Then, we demonstrated that exosomes from a highly liver metastatic colorectal cancer cell line (HT-29) could significantly increase the metastatic tumor burden and distribution in the mouse liver of Caco-2 colorectal cancer cells, which ordinarily exhibit poor liver metastatic potential. We further investigated the mechanisms by which HT-29-derivedexosomes influence the liver metastasis of colorectal cancer and found that mice treated with HT-29-derived exosomes had a relatively higher level of CXCR4 in the metastatic microenvironment, indicating that exosomes may promote colorectal cancer metastasis by recruiting CXCR4-expressing stromal cells to develop a permissive metastatic microenviron-
\end{abstract}

Correspondence to: Dr Suzhen Dong and Dr Lei Yu, Institute for Advanced Interdisciplinary Research, East China Normal University, 3663 Zhongshan Road, Shanghai 200062, P.R. China E-mail: szdong@brain.ecnu.edu.cn

E-mail: yulei@nbic.ecnu.edu.cn

Abbreviations: CRC, colorectal cancer; HCC, human colon cancer; qRT-PCR, quantitative reverse transcriptase-PCR; H\&E, hematoxylin and eosin; FBS, fetal bovine serum; PFA, paraformaldehyde; PBS, phosphate-buffer saline; PVDF, polyvinylidene difluoride; FACS, flow cytometry; HBSS, Hank's balanced salt solution; HRP, horseradish peroxidase; DAB, diaminobenzidine; EXO(HT-29), exosomes derived from HT-29 cells; EXO(Caco-2), exosomes derived from Caco- 2 cells

Key words: exosomes, colorectal cancer, liver metastasis, CXCR4, migration ment. Finally, the migration of Caco-2 cells was significantly increased following treatment with HT-29-derived exosomes in vitro, further supporting a role for exosomes in modulating colorectal tumor-derived liver metastasis. The data from the present study may facilitate further translational medicine research into the prevention and treatment of colorectal cancer liver metastasis.

\section{Introduction}

Colorectal cancer (CRC) is a major cause of patient morbidity and mortality worldwide. More than 1.2 million new cases of colorectal cancer occur every year globally, accounting for $10 \%$ of all cancer cases (1). In the clinic, the liver is the most common site for human colon cancer metastasis, and patients with untreated liver metastases have a median survival of only 5-10 months. Surgical resection remains the only known effective intervention for liver metastasis. However, the 5-year survival rates of patients treated by this method are just $30-50 \%$. Therefore, it is necessary to understand the mechanisms of liver metastasis of CRC, so that it can be prevented or significantly delayed.

Previous studies have shown that CRC metastasis is related to the communication between tumor cells and bone marrowderived hematopoietic progenitor cells via secreted factors such as chemokines and cytokines. These signals create a microenvironment that is suitable for the homing and proliferation of tumor cells (2). Recently, it has been revealed that a type of tumor-derived small membrane vesicles (also known as exosomes) are actively involved in pre-metastatic niche formation and metastasis (3-5). Exosomes, which are secreted by a variety of cell types of endosomal origin, are small membrane vesicles ranging from 30 to $150 \mathrm{~nm}$ in diameter. Exosomes participate in cell communication by transferring proteins and genetic materials (e.g., mRNAs and microRNAs) between cells (6-8). Originally, exosomes were considered as organelles with the function of removing intracellular debris, but in 1996, exosomes were shown to be involved in immunological 
processes (9). Since then, an increasing number of studies have focused on exosomes, and as the constituents of exosomes and their biological functions have become clearer, exosomes have attracted growing interest.

During the previous decade, a number of studies have shown that exosomes are functional in modulating the growth and metastasis of melanoma, breast cancer, glioblastoma and pancreatic adenocarcinoma $(5,10-13)$. It has been shown that exosomes play important roles in the epithelial-tomesenchymal transition, cancer stemness, programmed cell death, apoptosis, angiogenesis and metastasis $(14,15)$. Studies on melanoma have demonstrated that exosomes from highly metastatic melanomas can increase the metastatic behavior of primary tumors by permanently 'educating' bone marrow progenitors, inducing vascular leakiness at pre-metastatic sites, and reprogramming bone marrow progenitors toward a pro-vasculogenic phenotype (4). Other studies have also supported that exosomes are key regulators of the invasion and metastasis of melanomas $(10,11,16,17)$, and similarly, that breast cancer cells secrete exosomes into the metastatic microenvironment to promote the initiation of metastasis by transferring miRNAs to target cells (5). The mechanisms of metastasis differ between cancer types, and so the underlying detail of how exosomes regulate the metastasis of different malignancies is likely to vary. Studies employing mRNA and protein expression profiling techniques have shown that CRC cell-derived exosomes contain many microRNAs or proteins associated with oncogenes or metastasis-related genes (18-25) suggesting that exosomes might be related to liver metastasis of CRC. Nevertheless, no direct evidence for exosomes promoting CRC metastasis has previously been provided, and the present study aimed to explore the potential role of exosomes in promoting CRC liver metastasis.

Metastasis is a process of several sequential, interrelated steps, and represents a highly organized, non-random and organ-selective pattern $(26,27)$. A wide variety of molecules, including cytokines, chemokines and their receptors, and growth factors, have been implicated in the promotion of metastasis (28-32). An accumulation of evidence suggests that tumor metastasis is associated with the SDF-1/CXCR4 axis in many types of cancers (33-37), but the connection between exosomes and CXCR4 has not been evaluated. In the present study, we demonstrated the potential involvement of the SDF-1/CXCR4 signaling pathway in the process of exosomemediated metastasis of CRC cells.

Metastasis, i.e., the spread of cells from the primary neoplasm to distant organs and their relentless growth into secondary tumors, is a complex process including two major phases: firstly, translocation of a cancer cell from the primary tumor to a distant tissue, and secondly, colonization of the destination tissue. Migration is a key step for tumor cells to invade and metastasize to distant sites. In the present study, we illustrated that $\mathrm{CRC}$-derived exosomes can promote the migration of cancer cells.

We found that exosomes derived from HT-29 CRC cells play important roles in promoting colorectal tumor liver metastasis. This study is the first to report that HT-29-derived exosomes directly promoted the liver metastasis of Caco-2 cells, indicating a pivotal role for exosomes in regulating the liver metastasis of CRC. The mechanisms of the HT-29-derived exosomes in promoting Caco-2 cell liver metastasis might be associated with the SDF-1/CXCR4 axis, and the effect of the exosomes in promoting tumor cell migration may influence the increased CRC liver metastasis. The data from this study may provide useful information to assist future translational medicine studies in identifying new approaches for the prevention and treatment of colorectal cancer liver metastasis.

\section{Materials and methods}

Mice. Male Balb/c nude mice were obtained from Shanghai SLAC Laboratory Animal Co., Ltd. (Shanghai, China). Mice were housed in specific pathogen-free conditions and experiments were conducted in accordance with the guidelines of the Animals Act, 2014 (China) and approved by the Institutional Animal Care and Use Committee (IACUC approval ID \#M08022) of the East China Normal University.

Cells. The HT-29 and Caco-2 cells were obtained from the Type Culture Collection of the Chinese Academy of Sciences, Shanghai, China. The HT-29 cells were maintained in McCoy's $5 \mathrm{~A}$ medium with $10 \%$ (v/v) fetal bovine serum (FBS), and the Caco-2 cells were cultured in minimum essential medium (MEM) with $20 \%$ (v/v) FBS and $2 \mathrm{mmol} / \mathrm{l}$ glutamine (Life Technologies, Carlsbad, CA, USA; cat. no. 25030-018) in a humidified $5 \% \mathrm{CO}_{2}$ water-jacketed incubator.

Exosome purification and characterization. Cells were cultured in media supplemented with exosome-depleted FBS. FBS was depleted of bovine exosomes by ultracentrifugation at $100,000 \mathrm{x} g$ for $70 \mathrm{~min}$. Supernatant fractions were collected from the cell cultures after a 24 - to 48 -h incubation after pelleting the cells by centrifugation at $2000 \mathrm{x} \mathrm{g}$ for $30 \mathrm{~min}$. Exosomes were then harvested from the supernatants using total exosome isolation reagent (from cell culture media) (Life Technologies; cat no. 4478359) according to the manufacturer's instructions. Exosomal proteins were isolated with a total exosome RNA and protein isolation kit (Life Technologies; cat no. 4478545). The concentrations of isolated proteins were quantified by bicinchoninic acid assay (Beyotime, Beijing, China; cat. no. P0010S).

Electron microscopy. Exosomes purified as described above were fixed in $2 \%(\mathrm{w} / \mathrm{v})$ paraformaldehyde (PFA) in $200 \mathrm{mM}$ phosphate-buffered saline (PBS) (pH 7.4). Fixed exosomes were dropped onto a Formvar/carbon-coated grid and left to dry at room temperature for $20 \mathrm{~min}$. After washing in PBS, the exosomes were fixed in $1 \%(\mathrm{w} / \mathrm{v})$ glutaraldehyde for $5 \mathrm{~min}$, washed in water, and stained with saturated aqueous uranyl oxalate for $5 \mathrm{~min}$. Samples were then embedded in a solution of $0.4 \%(\mathrm{w} / \mathrm{v})$ uranyl acetate and $1.8 \%(\mathrm{w} / \mathrm{v})$ methylcellulose and incubated on ice for $10 \mathrm{~min}$. The excess liquid was then removed. The grid was dried at room temperature for $10 \mathrm{~min}$ and viewed using an electron microscope (JEM-2100; JEOL, Tokyo, Japan) (38).

Western blot analysis. Exosomal proteins (isolated as described above) were resolved by sodium dodecyl sulfate polyacrylamide gel electrophoresis (SDS-PAGE) and transferred to polyvinylidene difluoride (PVDF) membrane (Millipore, 
Billerica, MA, USA; cat. no. IPVH00010) at $100 \mathrm{~V}$ for $45 \mathrm{~min}$. After blocking in Tris-buffered saline containing $0.05 \%(\mathrm{v} / \mathrm{v})$ Tween-20 (TBST) containing 5\% (w/v) skimmed milk powder at room temperature for $2 \mathrm{~h}$, the membranes were incubated with primary antibodies specific to heat-shock protein (HSP)70 (BD Biosciences, San Jose, CA, USA; cat. no. 610607; 1:2000 dilution) and cluster of differentiation (CD)63 (Abcam, Cambridge, UK; cat no. ab8219; 1:200 dilution) overnight at $4^{\circ} \mathrm{C}$. Antibodies to $\beta$-actin (Sigma-Aldrich, St. Louis, MO, USA; cat no. A1978; 1:1000 dilution) were used as loading controls. The membranes were then rinsed three times with TBST and incubated with goat anti-mouse $\mathrm{IgG}$ at a dilution of 1:2,000 at room temperature for $90 \mathrm{~min}$. After three final washes with TBST, the membranes were developed using an enhanced chemiluminescence kit (Beyotime; cat. no. P0018).

Flow cytometry (FACS). For FACS analysis, exosomes were coated onto 4-mm-diameter aldehyde/sulfate latex beads (Life Technologies; cat. no. A37304), as individual exosomes are too small to be visualized by FACS. Briefly, $30 \mu \mathrm{g}$ exosomes were incubated with $100 \mu \mathrm{l}$ 4-mm-diameter aldehyde/sulfate latex beads for $15 \mathrm{~min}$ at room temperature. The mixture was then transferred to $900 \mu \mathrm{l}$ of PBS and incubated at room temperature for a further $2 \mathrm{~h}$. The coupling reaction was terminated by the addition of $100 \mathrm{mM}$ glycine. Exosome-coated beads were stained with primary mouse monoclonal antibodies directed against CD63, washed twice, incubated with Alexa Fluor ${ }^{\circledR}$ 488-labeled secondary antibody in darkness for $60 \mathrm{~min}$, and analyzed by flow cytometry (guava easyCyte ${ }^{\mathrm{TM}} \mathrm{HT}$; Millipore).

Metastasis and liver colonization studies. To develop a mouse model of hepatic metastasis, tumor cells were implanted into the spleens of nude mice. HT-29 or Caco- 2 cells $\left[1 \times 10^{6}\right.$ cells in $100 \mu$ l Hank's balanced salt solution (HBSS)] were injected into the spleen of 6-week-old Balb/c male mice, with 10 animals per treatment group. The mice were sacrificed 8 weeks after injection of the tumor cells, and liver metastases were enumerated immediately. To analyze the role of exosomes in tumor metastasis, 6-week-old Balb/c nude male mice were injected intrasplenically with $1 \times 10^{6}$ Caco- 2 cells. After the implantation of Caco- 2 cells, $10 \mu \mathrm{g}$ HT-29-derived exosomes were intravenously injected every three days for 28 days. We injected equal and physiological concentrations of Caco-2-derived exosomes intravenously into the control mice. Each group contained 6-8 mice. Autopsies were performed after 14 weeks and the presence of metastases was examined macroscopically. All liver samples were dissected and divided into two parts: one part was frozen for RNA extraction and quantitative reverse transcriptase-PCR (qRT-PCR) assay, while the other part was fixed in $4 \%$ (w/v) PFA for histologic analysis. The tissues used to evaluate metastasis in the liver and the metastatic microenvironment were paraffin embedded and cut into slices. The sections were stained with hematoxylin and eosin or immunohistochemically stained, and metastases were evaluated by inverted microscopy (IX71; Olympus, Tokyo, Japan).

Quantitative reverse transcriptase-PCR. Total RNA was extracted from the frozen liver tissue from each mouse using TRIzol $^{\circledR}$ (Life Technologies; cat no. 10296010). The RNA samples were used to generate cDNA using Moloney murine leukemia virus reverse transcriptase (Takara; cat. no. D2639A). The cDNA samples were used as templates for SYBR ${ }^{\circledR}$ Green qPCR (ABI7500; Applied Biosystems, Foster City, CA, USA). The primer pairs for the genes were designed using Beacon Designer 8 software (Premier Biosoft, Palo Alto, CA, USA) and synthesized by Life Technology. The oligonucleotide primer sequences were as follows: 5'-GTAGAACTGTAGAG GAAGAA-3' (sense) and 5'-CGGAATGAAGAGATTAT GC-3' (antisense) for CXCR4; 5'-AACAGACAGAGTAAG GAA-3' (sense) and 5'-CTAACAATTCTACAGAGTATTC-3' (antisense) for SDF-1; 5'-GGCTGTATTCCCCTCCATCG-3' (sense) and 5'-CCAGTTGGTAACAATGCCATGT-3' (antisense) for $\beta$-actin. $\beta$-actin was used as an endogenous reference. Gene expression levels were calculated as $2^{-\Delta \Delta \mathrm{Ct}}$ values.

Immunohistochemistry. Immunohistochemistry was performed to examine the expression of CXCR4 around the liver metastatic niche. The sections of surgical specimens were deparaffinized with xylene and rehydrated with ethanol. Antigen retrieval was performed by keeping the sections in $10 \mathrm{mM}$ sodium citrate buffer ( $\mathrm{pH} 6.0$ ) at $92-98^{\circ} \mathrm{C}$ for $20 \mathrm{~min}$. After cooling for $20 \mathrm{~min}$, the sections were rinsed with PBS. Slides were incubated with $3 \% \mathrm{H}_{2} \mathrm{O}_{2}$ and rinsed in $0.1 \mathrm{M}$ PBS (pH 7.4), then treated with blocking solution (5\% BSA in $0.1 \mathrm{M}$ PBS) for $1 \mathrm{~h}$ at room temperature and incubated with the CXCR4 primary antibody [Santa Cruz Biotechnology, Dallas, TX, USA; cat. no. sc-9046; 1:50 dilution in 0.1 M PBS, with $1 \%(\mathrm{w} / \mathrm{v}) \mathrm{BSA}$ ] overnight at $4^{\circ} \mathrm{C}$. After rinsing consecutively in PBS, the sections were incubated with horseradish peroxidase (HRP)-labeled goat anti-rabbit secondary antibody (1:150 dilution) at $37^{\circ} \mathrm{C}$ for $10 \mathrm{~min}$. The immunoreactivity was visualized with the 3,3'-diaminobenzidine tetrachloride (DAB) solution from a HRP-polymer anti-rabbit IHC kit (Maixin Biology, Fuzhou, China; cat. no. KIT-5010) according to the manufacturer's protocol. The nuclei were counterstained with hematoxylin. Sections were viewed by inverted microscopy and photographed (IX71; Olympus).

Migration assay. The migration assay was performed in 24-well Transwell ${ }^{\circledR}$ plates (Corning, New York, NY, USA; cat. no. 3422) using inserts with $8-\mu \mathrm{m}$ pore size membranes. To investigate the role of exosomes in cell migration, Caco-2 cells were co-cultured with exosomes derived from HT-29 or Caco- 2 cells before being seeded onto the top chamber. Caco- 2 cells were suspended at $1 \times 10^{6}$ cells $/ \mathrm{ml}$ and mixed with $10 \mu \mathrm{g} / \mathrm{ml}$ exosomes in Dulbecco's modified Eagle's medium (DMEM) without FBS, then added to the upper chambers, and the same medium containing $20 \%$ FBS was placed in the lower chamber. Blank controls without exosomes were set up at the same time. After $36 \mathrm{~h}$, cells on the lower membrane were stained with crystal violet (Beyotime; cat no. C0121) for 15 min and counted under a light microscope in at least five different fields (original magnification, x200). Each clone was plated in triplicate in each experiment.

\section{Results}

Construction of the CRC liver metastasis animal model with HT-29 and Caco-2 cells. It has been demonstrated that HT-29 

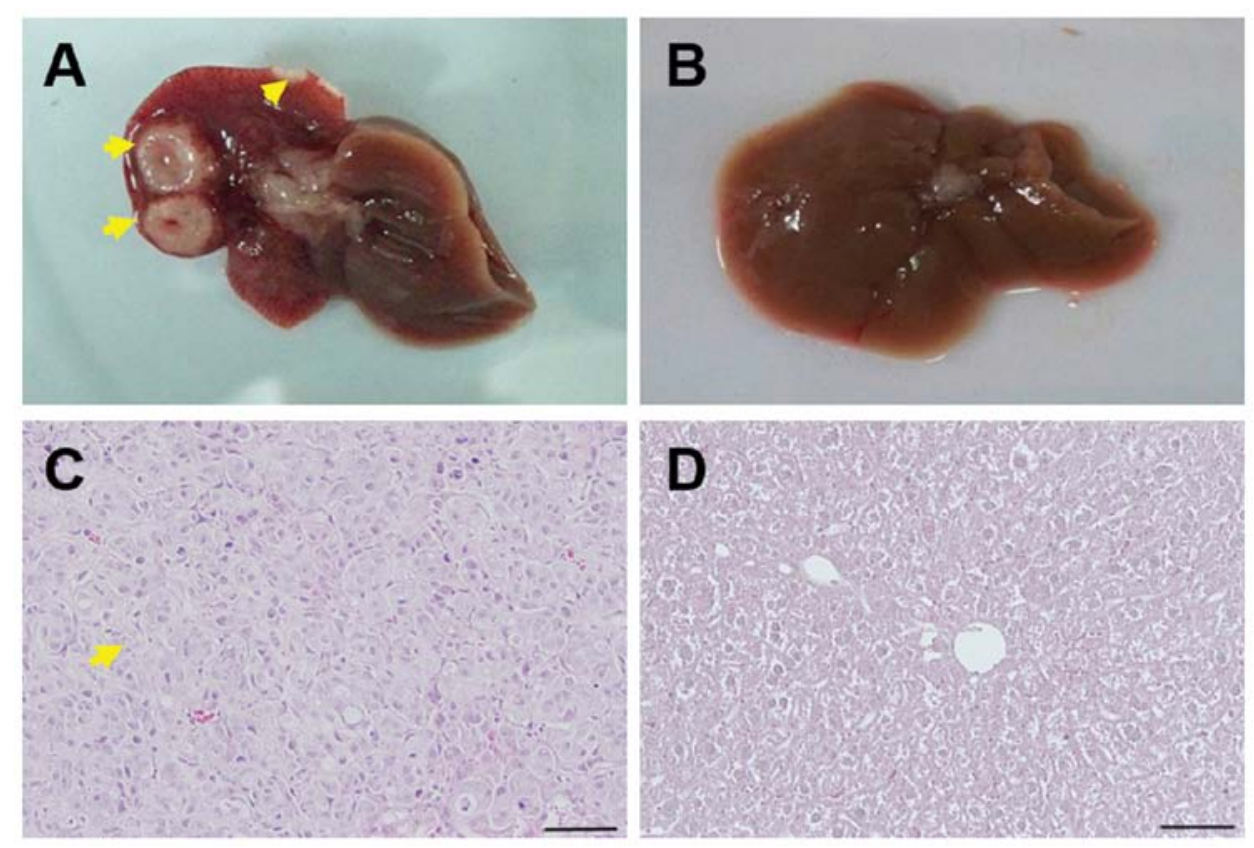

Figure 1. Evident liver metastasis is induced by HT-29 cells, but not Caco-2 cells. HT-29 or Caco-2 cells were inoculated into nude mice by intrasplenic injection. The mice were sacrificed 8 weeks later, and liver metastases were enumerated immediately, without prior fixation. Representative livers from (A) HT-29- or (B) Caco-2-seeded mice are shown. Representative H\&E staining results of liver sections from (C) HT-29- or (D) Caco-2-seeded mice. Arrows indicate tumor nodules. Scale bar, $100 \mu \mathrm{m}$.

cells are highly metastatic to the liver, while Caco-2 cells do not induce liver metastasis when intrasplenically injected into nude mice $(39,40)$. To confirm these prior reports, $10^{6} \mathrm{HT}-29$ or Caco-2 cells were inoculated into 6-week old male nude mice by intrasplenic injection $(39,41)$. In agreement with the results of previous studies, after 8 weeks, all of the 10 mice injected with HT-29 cells bore tumors in the liver (Fig. 1A). However, of the 10 mice injected with Caco-2 cells, no mice developed tumors in the liver (Fig. 1B). Furthermore, the results of hematoxylin and eosin (H\&E) staining also showed evidence for a metastatic niche in sections from the HT-29-seeded mice livers, whereas no evidence was noted for a metastatic niche in sections from the livers of the Caco-2-seeded mice (Fig. 1C and D).

Characterization of the exosomes derived from HT-29 and Caco-2 colorectal cancer cells. To investigate the role of exosomes in CRC liver metastasis, exosomes were isolated from the cell culture media of HT-29 and Caco-2 cells, and their identity was confirmed by electron microscopy, western blotting and flow cytometry. The vesicles isolated from the HT-29 cells appeared as round-shaped 60-100 nm diameter vesicles under electron microscopy (Fig. 2A), whereas culture media from the Caco-2 cells contained a heterogeneous population of vesicles comprising both round-shaped 40-80 nm diameter vesicles, as well as larger vesicles $\sim 80-120 \mathrm{~nm}$ in diameter (Fig. 2B), consistent with exosomes $(8,42)$. We further confirmed whether these vesicles were exosomes by performing western blotting with antibodies against two commonly used exosomal markers, the tetraspanin molecule CD63 and HSP70 $(8,38,43)$. The exosomes isolated from the cell culture media of both cell types contained CD63 and HSP70 at high levels (Fig. 2C). Results of flow cytometry also showed CD63 to be present in exosomes from HT-29 and Caco-2 cells (Fig. 2D and E) (7).

Investigation of the effect of HT-29-derived exosomes on Caco-2 cell liver metastasis. To examine the role of tumor-derived exosomes in CRC liver metastasis, HT-29- or Caco-2-derived exosomes were administered to mice by intravenous injection, starting 5 days after the intrasplenic injection of Caco-2 cells. Only $57 \%$ of the mice treated with exosomes derived from Caco-2 cells showed liver metastasis at 14 weeks after the tumor cells were injected, and there were only small tumor nodules on the surfaces of the livers (4/7 mice) (Fig. 3B). However, all the mice treated with exosomes from the HT-29 cells showed evident liver metastasis and had a greater metastatic burden in the liver $(8 / 8$ mice) compared with the mice treated with exosomes derived from the poorly metastatic Caco-2 cells (Fig. 3A). H\&E staining also supported these results (Fig. 3C and D). These findings strongly indicate that exosomes from highly metastatic CRC cells could enhance the metastatic capability of poorly metastatic CRC cells.

Putative mechanisms of HT-29-derived exosomes on promoting Caco-2 cell liver metastasis: Investigation of the SDF-1 $\alpha / C X C R 4$ axis. The SDF- $1 \alpha / \mathrm{CXCR} 4$ axis plays important roles in many types of malignancies, including lung, brain, prostate and breast cancers $(35,36)$. We speculated that exosomes might regulate tumor metastasis through this signaling pathway. To investigate this hypothesis, the expression levels of SDF- $1 \alpha$ and CXCR4 in the metastatic microenvironment of the liver tissues of the animal models were analyzed by quantitative reverse transcriptase-PCR (qRTPCR) and immunohistochemistry. The CXCR4 mRNA level in 

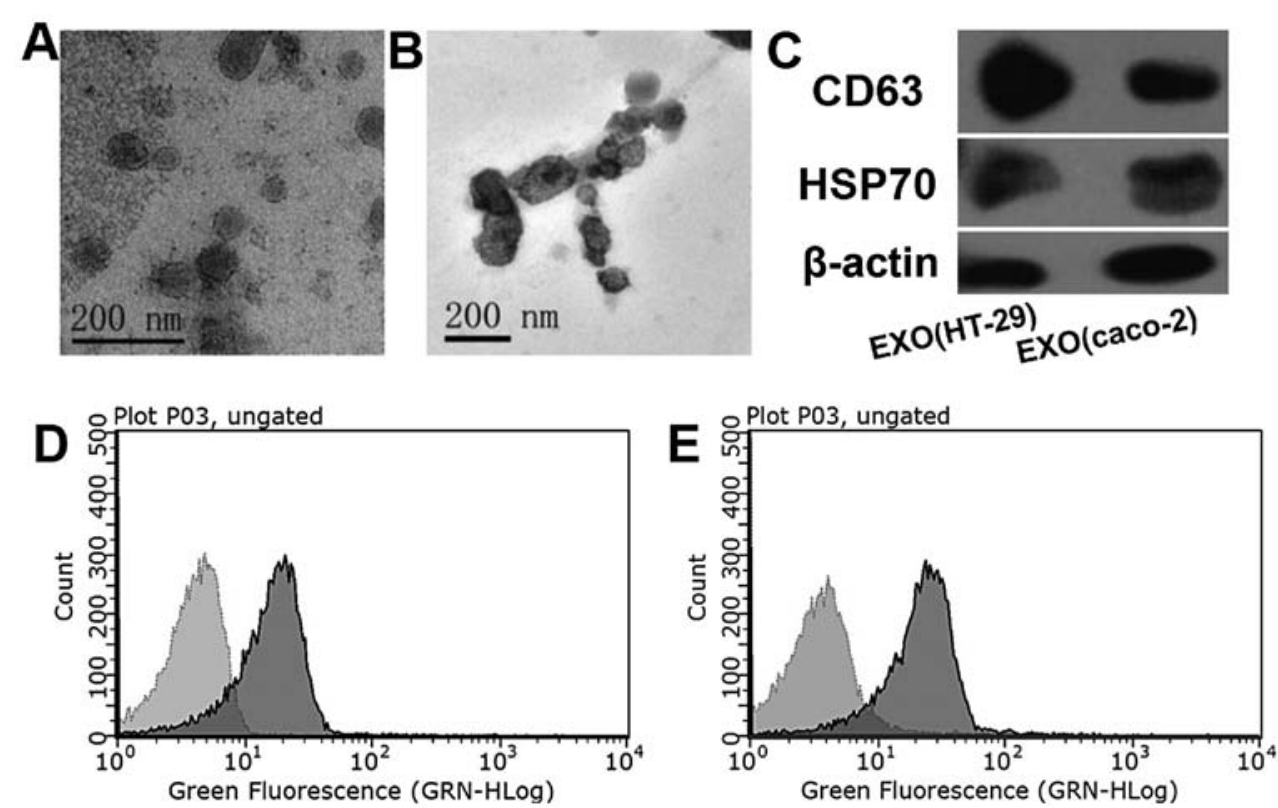

Figure 2. Characterization of exosomes derived from HT-29 and Caco-2 cells. (A and B) Electron microscopic images of exosomes from HT-29 and Caco-2 cells, respectively, which revealed that the isolated exosomes comprised round-shaped 30-150 nm diameter vesicles. (C) Western blot analysis of two marker proteins of exosomes, CD63 and HSP70. In the (D) HT-29 and (E) Caco-2 cells, the membranous CD63 expression of exosomes was detected by flow cytometry. $\mathrm{EXO}(\mathrm{HT}-29)$, exosomes derived from HT-29 cells. $\mathrm{EXO}(\mathrm{Caco}-2)$, exosomes derived from Caco-2 cells.
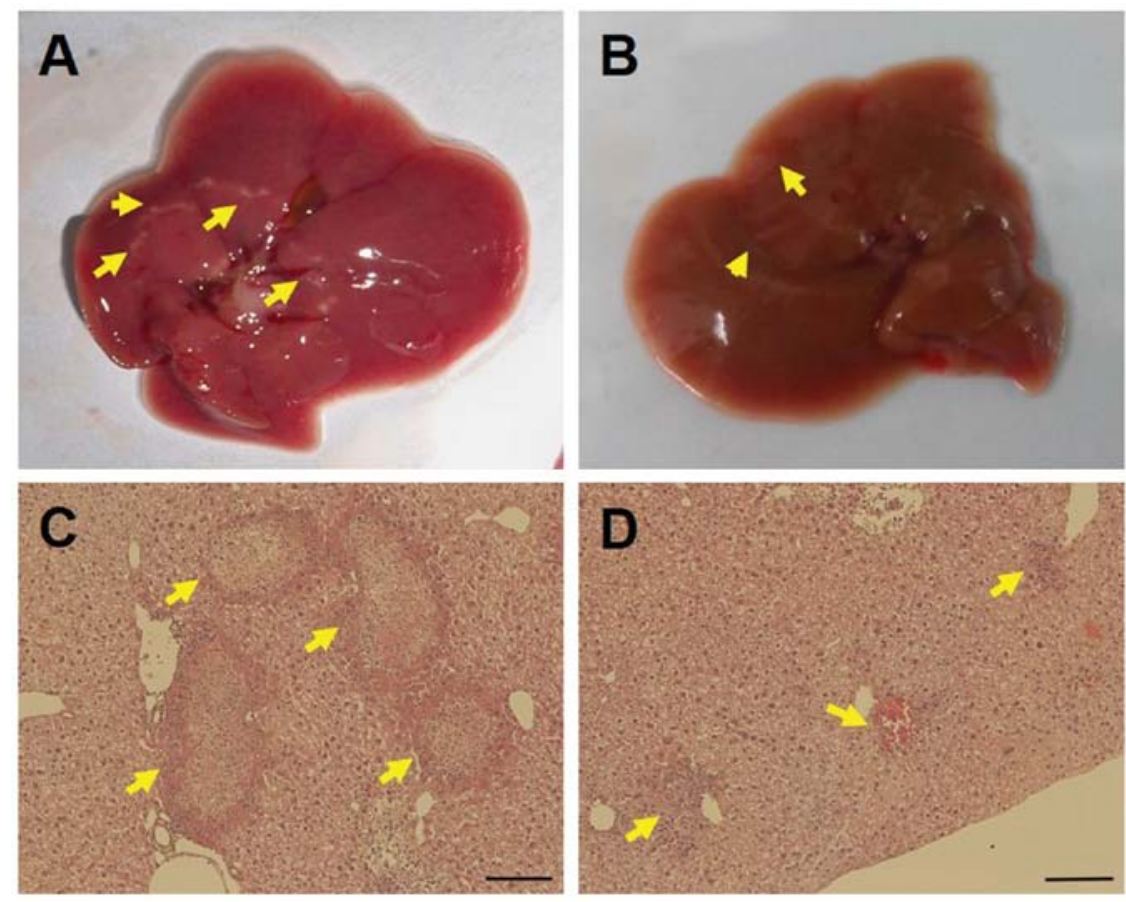

Figure 3. Exosomes derived from HT-29 cells promote liver metastasis. HT-29-derived or Caco-2-derived exosomes (10 $\mu \mathrm{g})$ were intravenously injected in the mice every three days for 1 month after intrasplenic injections of Caco-2 cells. Autopsies were performed after 14 weeks and the presence of metastases was examined macroscopically. (A and B) Representative livers from (A) HT-29-derived and (B) Caco-2-derived exosome-treated Caco-2-implanted mice are shown. (C and D) Representative H\&E staining results of the liver slices from (C) HT-29-derived and (D) Caco-2-derived exosome-treated Caco-2-implanted mice. Arrows indicate tumor nodules. Scale bar, $50 \mu \mathrm{m}$.

the liver of the HT-29-implanted mice was significantly higher than that of the Caco-2-implanted mice (Fig. 4A, P<0.05), which is consistent with the results of immunohistochemistry (Fig. 4E and F). Treatment with HT-29-derived exosomes led to an increase in the expression level of CXCR4 in the liver of the Caco-2-implanted mice compared with those treated with the Caco-2-derived exosomes (Fig. 4C, $\mathrm{P}=0.15$ ). The results of immunohistochemistry also revealed that CXCR4 showed a higher protein level in the liver of the HT-29-derived exosome-treated Caco-2-implanted mice, in comparison with 

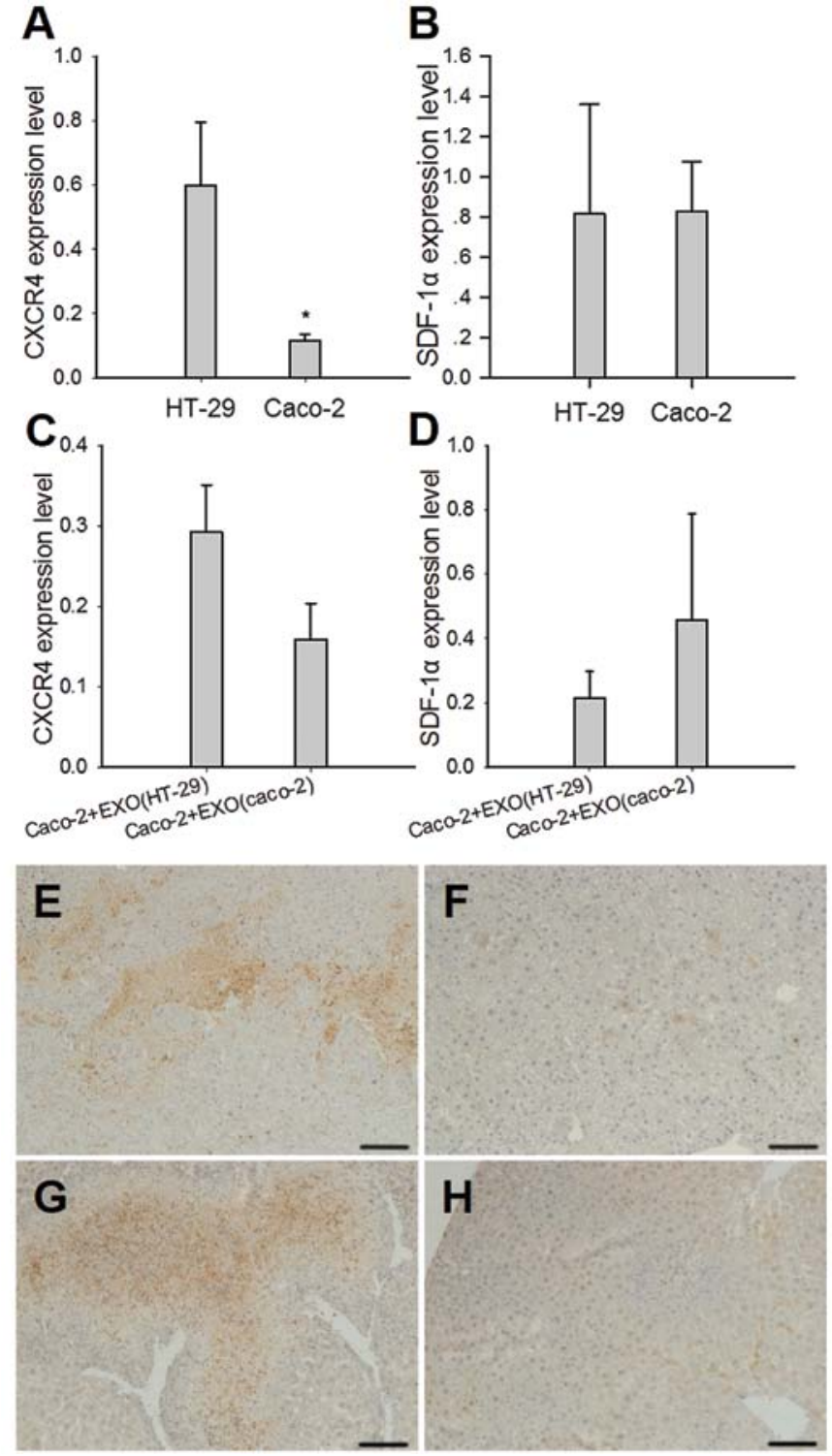

Figure 4. Exosomes derived from HT-29 cells enhance CXCR4 expression in the liver. The relative mRNA levels of (A and C) CXCR4 and (B and D) SDF-1 $\alpha$ were measured by qRT-PCR in the livers of HT-29- or Caco-2implanted mice ( $\mathrm{A}$ and $\mathrm{B} ; \mathrm{n}=5$ ), or Caco-2-implanted mice with injections of HT-29- or Caco-2-derived exosomes ( $\mathrm{C}$ and $\mathrm{D} ; \mathrm{n}=3$ ). Relative expression levels were calculated as the ratio of the target gene expression level to the $\beta$-actin expression level in the same sample. Data are shown as the mean \pm SEM and analyzed using the Student's t-test; "P $<0.05$. (E-H) Representative images of immunohistochemistry results of liver sections derived from (E) HT-29- or (F) Caco-2-implanted mice, or Caco-2-implanted mice injected with $(\mathrm{G})$ HT-29- or (H) Caco-2-derived exosomes. Scale bar, $100 \mu \mathrm{m}$.

the Caco-2-implanted mice that were treated with the Caco-2derived exosomes (Fig. 4G and H). The expression of SDF-1 mRNA was also detected, but no significant differences in expression were observed between groups (Fig. 4B and D).

Effects of HT-29-derived exosomes on the migration ability of Caco-2 cells in vitro. Exosomes have previously been shown to mediate the migration of endothelial cells (44). We investigated whether HT-29-derived exosomes could affect the migration of Caco- 2 cells. In vitro cell migration assays were performed by treating Caco-2 cells with HT-29- or

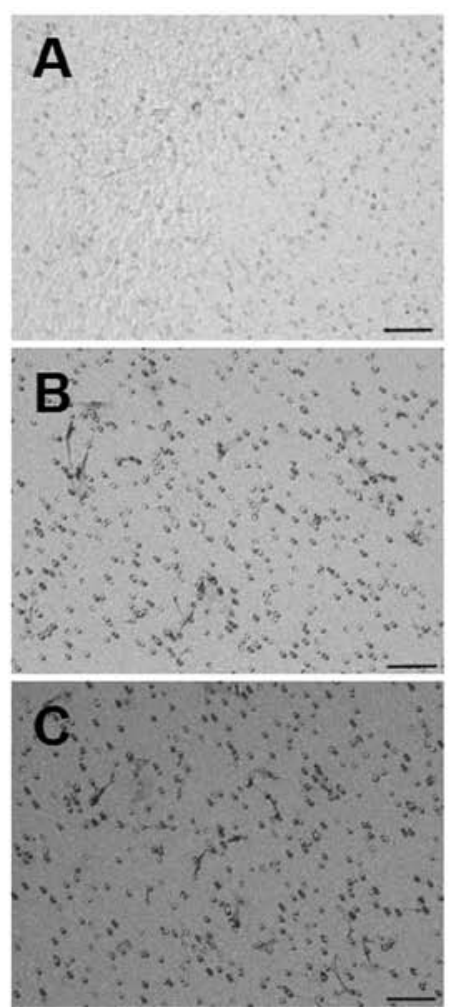

D

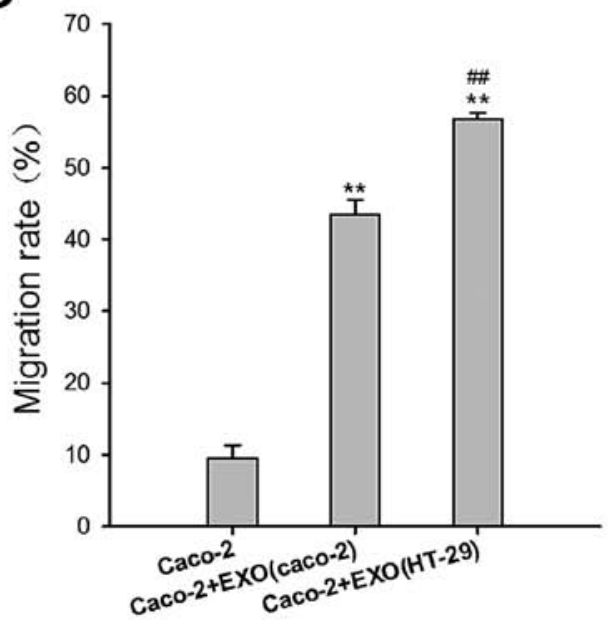

Figure 5. Exosomes increase the migration ability of Caco-2 cells in vitro. (A-C) The effect of exosomes on Caco-2 migration was determined by Transwell ${ }^{\boxplus}$ cell migration assay. Representative images at $36 \mathrm{~h}$ after seeding of Caco-2 cells (A) without external exosomes, (B) with Caco-2-derived exosomes or (C) with HT-29-derived exosomes are shown. Scale bar, $50 \mu \mathrm{m}$. (D) Quantification of the average number of migrated Caco-2 cells by treatment at $36 \mathrm{~h}$ after planting. Results are expressed as the mean $\pm \mathrm{SE} ;{ }^{* *} \mathrm{P}<0.01$, vs. Caco- 2 cells without external exosomes, ${ }^{\# \prime} \mathrm{P}<0.01$ vs. Caco-2 cells with Caco-2-derived exosomes.

Caco-2-derived exosomes. Caco-2 cells without treatment with external exosomes served as the experimental control. The results showed that exosomes from both the HT-29 and Caco-2 cells significantly enhanced the migration of Caco-2 cells and that a greater number of Caco-2 cells that were treated with the HT-29-derived exosomes migrated than did those that were treated with the Caco-2-derived exosomes (Fig. 5, P=0.004). 


\section{Discussion}

Liver metastasis is one of the main pathological developments that leads to the death of CRC patients. Accumulating evidence indicates that exosomes are key mediators of tumor metastasis in melanoma and breast cancer (10-12,45). Exosomes may play a role in the generation of metastatic microenvironments, but the underlying molecular and cellular mechanisms have not been determined. This study elucidated for the first time the roles of exosomes derived from HT-29, a highly liver metastatic CRC cell line, in promotion of liver metastasis by the poorly liver metastatic Caco-2 cell line.

To verify the direct role of exosomes in CRC metastasis, we established liver metastatic nude mouse models of CRC cells by intrasplenic injection of HT-29 and Caco-2 cells, separately, to examine the characteristics of these two cell lines in their frequency and severity of liver metastasis. It was previously reported that HT-29 cells are highly metastatic to the liver, in contrast to Caco- 2 cells, which have been reported to be poorly metastatic to the liver $(41,46)$. Our results were consistent with those of previous reports and showed that the HT-29 cells had a significantly higher potential to undergo liver metastasis than did the Caco-2 cells (Fig. 1). These initial findings laid the foundations for our further research. The findings were also consistent with previous results showing that HT-29 cells had a greater impact than Caco-2 cells on metastatic tumor growth after intrasplenic implantation into nude mice $(39,46,47)$. Subsequently, animal models were used to investigate the function of exosomes in liver metastasis. HT-29- or Caco-2-derived exosomes were extracted from cell culture media and characterized by electron microscopy, western blotting and flow cytometry (Fig. 2) and then administered to Caco-2-inoculated mice by intravenous injection to examine the effects of exosomes from highly metastatic CRC cells on the liver metastasis of the normally poorly liver metastatic Caco-2 cells. Treatment with HT-29-derived exosomes induced a pronounced enhancement of metastasis of Caco-2 cells (Fig. 3). These results showed that exosomes play a key role in colorectal cancer liver metastasis.

A multitude of evidence from other cancers including melanoma and breast cancer has demonstrated that exosomes can mediate tumor metastasis by the horizontal transfer of molecules such as proteins and microRNAs (48-50). The exosome-delivered miRNAs were found to mostly target metastasis-related pathways, such as proteases, adhesion molecules, chemokine ligands, and cell cycle- and angiogenesis-promoting genes. Studies on lung cancer and melanoma have revealed that exosomes are involved in regulating angiogenesis and epithelial-mesenchymal transition $(17,51)$. However, the role of exosomes in cancer metastasis may vary among cancer types, given that the established metastatic mechanisms of different cancers are also variable. Thus, the mechanisms by which exosomes participate in the regulation of CRC liver metastasis were then elucidated.

SDF-1 and its receptor CXCR4 have prominent roles in many types of malignancies, including lung, brain, prostate, and breast cancer as well as colorectal cancer $(35,36,52)$ and regulate the motility of cells, including tumor cells and MSCs $(32-34,37,53,54)$. Therefore, we suspected that exosomes might increase SDF-1 expression in the metastatic microen- vironment and thereby attract cancer cells and other stromal cells. However, no clear differences were detected in the SDF-1 expression level among the different animal models (HT-29- or Caco-2 cell-implanted mice and HT-29- or Caco-2-derived exosome-treated Caco-2-implanted mice) (Fig. 4B and D). On the other hand, measurement of the expression levels of CXCR4 in the livers of the HT-29 and Caco-2 xenograft models showed that the livers of the HT-29 xenograft model had significantly higher CXCR4 levels than those of the Caco-2 xenograft mouse model (Fig. 4A). Meanwhile, HT-29-derived exosomes induced higher CXCR4 expression levels than Caco-2-derived exosomes (Fig. 4C and E-H). The results indicated that HT-29-derived exosomes might affect CRC metastasis by increasing CXCR4 expression by many types of stromal cells and promoting the recruitment of these CXCR4-expressing stromal cells to develop a suitable metastatic microenvironment. However, the underlying mechanisms need further investigation.

Cancer metastasis is a complex process involving multiple steps, including invasion, angiogenesis, trafficking of cancer cells through blood vessels, extravasation, organ-specific homing and growth. Tumor cell migration is one of the most important steps of tumor metastasis. Whether HT-29derived exosomes can affect the migration of Caco-2 cells was examined by cell migration assay in vitro. Our results showed that exosomes from both HT-29 and Caco-2 cells promoted the migration of Caco-2 cells, and HT-29-derived exosomes had a greater impact on Caco- 2 cell migration than Caco-2-derived exosomes did (Fig. 5). Thus, exosomes might promote metastasis by affecting the migration of CRC cells.

In the present study, we demonstrated a pivotal role for exosomes in the liver metastasis of colorectal cancer, which may function by affecting cellular migration. We also showed that the effects of exosomes might be related to the creation of a CXCR4-enriched microenvironment, which is comprised of stromal cells including immune cells, endothelial cells, fibroblasts, bone marrow-derived cells and stem cells. Our research leads to the further understanding of how exosomes affect the liver metastasis of CRC and provide perspective regarding the therapeutic window for therapy of colorectal cancer. However, whether exosomes of CRC function similarly to those of melanoma, by promoting the 'education' and mobilization of bone-marrow derived cells or other stromal cells, and what components of exosomes are involved in the CXCR4 expression warrant further study.

\section{Acknowledgements}

The present study was supported by grants from the National Natural Science Foundation of China (81202471), the International Cooperation Project of the Science and Technology Commission of Shanghai Municipality (12520708000) and the Zhejiang Provincial Natural Science Foundation of China (Y14H300005).

\section{References}

1. Jemal A, Bray F, Center MM, Ferlay J, Ward E and Forman D: Global cancer statistics. CA Cancer J Clin 61: 69-90, 2011.

2. Pancione M, Giordano G, Remo A, et al: Immune escape mechanisms in colorectal cancer pathogenesis and liver metastasis. J Immunol Res 2014: 11, 2014. 
3. Alderton GK: Metastasis. Exosomes drive premetastatic niche formation. Nat Rev Cancer 12: 447, 2012.

4. Peinado H, Aleckovic M, Lavotshkin S, et al: Melanoma exosomes educate bone marrow progenitor cells toward a pro-metastatic phenotype through MET. Nat Med 18: 883-891, 2012.

5. Kosaka N, Iguchi H, Hagiwara K, Yoshioka Y, Takeshita F and Ochiya T: Neutral sphingomyelinase 2 (nSMase2)-dependent exosomal transfer of angiogenic microRNAs regulate cancer cel metastasis. J Biol Chem 288: 10849-10859, 2013.

6. Thery C, Ostrowski M and Segura E: Membrane vesicles as conveyors of immune responses. Nat Rev Immunol 9: 581-593, 2009.

7. Valadi H, Ekstrom K, Bossios A, Sjostrand M, Lee JJ and Lotvall JO: Exosome-mediated transfer of mRNAs and microRNAs is a novel mechanism of genetic exchange between cells. Nat Cell Biol 9: 654-659, 2007.

8. Bang $\mathrm{C}$ and Thum T: Exosomes: new players in cell-cell communication. Int J Biochem Cell Biol 44: 2060-2064, 2012.

9. Raposo G, Nijman HW, Stoorvogel W, et al: B lymphocytes secrete antigen-presenting vesicles. J Exp Med 183: 1161-1172, 1996.

10. Lima LG, Chammas R, Monteiro RQ, Moreira ME and Barcinski MA: Tumor-derived microvesicles modulate the establishment of metastatic melanoma in a phosphatidylserinedependent manner. Cancer Lett 283: 168-175, 2009.

11. Hood JL, San RS and Wickline SA: Exosomes released by melanoma cells prepare sentinel lymph nodes for tumor metastasis. Cancer Res 71: 3792-3801, 2011.

12. Skog J, Wurdinger T, van Rijn S, et al: Glioblastoma microvesicles transport RNA and proteins that promote tumour growth and provide diagnostic biomarkers. Nat Cell Biol 10: 1470-1476, 2008.

13. Jung T, Castellana D, Klingbeil P, et al: CD44v6 dependence of premetastatic niche preparation by exosomes. Neoplasia 11: 1093-1105, 2009

14. Azmi AS, Bao B and Sarkar FH: Exosomes in cancer development, metastasis, and drug resistance: a comprehensive review. Cancer Metastasis Rev 32: 623-642, 2013.

15. Vlassov AV, Magdaleno S, Setterquist R and Conrad R Exosomes: current knowledge of their composition, biological functions, and diagnostic and therapeutic potentials. Biochim Biophys Acta 1820: 940-948, 2012.

16. Liu Y, Xiang X, Zhuang X, et al: Contribution of MyD88 to the tumor exosome-mediated induction of myeloid derived suppressor cells. Am J Pathol 176: 2490-2499, 2010.

17. Hood JL, Pan H, Lanza GM and Wickline SA: Paracrine induction of endothelium by tumor exosomes. Lab Invest 89 : $1317-1328,2009$.

18. Silva J, Garcia V, Rodriguez M, et al: Analysis of exosome release and its prognostic value in human colorectal cancer. Genes Chromosomes Cancer 51: 409-418, 2012

19. Hong B, Cho JH, Kim H, et al: Colorectal cancer cell-derived microvesicles are enriched in cell cycle-related mRNAs that promote proliferation of endothelial cells. BMC Genomics 10: $556,2009$.

20. Choi DS, Lee JM, Park GW, et al: Proteomic analysis of microvesicles derived from human colorectal cancer cells. J Proteome Res 6: 4646-4655, 2007.

21. Mathivanan S, Lim JW, Tauro BJ, Ji H, Moritz RL and Simpson RJ: Proteomics analysis of A33 immunoaffinitypurified exosomes released from the human colon tumor cell line LIM1215 reveals a tissue-specific protein signature. Mol Cell Proteomics 9: 197-208, 2010.

22. Choi DS, Park JO, Jang SC, et al: Proteomic analysis of microvesicles derived from human colorectal cancer ascites. Proteomics 11: 2745-2751, 2011

23. Demory Beckler M, Higginbotham JN, Franklin JL, et al: Proteomic analysis of exosomes from mutant KRAS colon cancer cells identifies intercellular transfer of mutant KRAS Mol Cell Proteomics 12: 343-355, 2012.

24. Choi DS, Choi DY, Hong BS, et al: Quantitative proteomics of extracellular vesicles derived from human primary and metastatic colorectal cancer cells. J Extracell Vesicles 1: 18704, 2012. doi: http://dx.doi.org/10.3402/jev.vli0.18704.

25. Ji H, Greening DW, Barnes TW, et al: Proteome profiling of exosomes derived from human primary and metastatic colorectal cancer cells reveal differential expression of key metastatic factors and signal transduction components. Proteomics 13 1672-1686, 2013.
26. Nicolson GL: Paracrine and autocrine growth mechanisms in tumor metastasis to specific sites with particular emphasis on brain and lung metastasis. Cancer Metastasis Rev 12: 325-343, 1993.

27. Fidler IJ: The pathogenesis of cancer metastasis: the 'seed and soil' hypothesis revisited. Nat Rev Cancer 3: 453-458, 2003.

28. Joukov V, Pajusola K, Kaipainen A, et al: A novel vascular endothelial growth factor, VEGF-C, is a ligand for the Flt4 (VEGFR-3) and KDR (VEGFR-2) receptor tyrosine kinases. EMBO J 15: 1751, 1996

29. Achen MG, Jeltsch M, Kukk E, et al: Vascular endothelial growth factor D (VEGF-D) is a ligand for the tyrosine kinases VEGF receptor 2 (Flk1) and VEGF receptor 3 (Flt4). Proc Natl Acad Sci USA 95: 548-553, 1998

30. Youngs SJ, Ali SA, Taub DD and Rees RC: Chemokines induce migrational responses in human breast carcinoma cell lines. Int J Cancer 71: 257-266, 1997.

31. Kim H and Muller WJ: The role of the epidermal growth factor receptor family in mammary tumorigenesis and metastasis. Exp Cell Res 253: 78-87, 1999.

32. Muller A, Homey B, Soto H, et al: Involvement of chemokine receptors in breast cancer metastasis. Nature 410: 50-56, 2001.

33. Zeelenberg IS, Ruuls-Van Stalle L and Roos E: Retention of CXCR4 in the endoplasmic reticulum blocks dissemination of a T cell hybridoma. J Clin Invest 108: 269-277, 2001.

34. Kitaori T, Ito H, Schwarz EM, et al: Stromal cell-derived factor $1 /$ CXCR4 signaling is critical for the recruitment of mesenchymal stem cells to the fracture site during skeletal repair in a mouse model. Arthritis Rheum 60: 813-823, 2009.

35. Dewan MZ, Ahmed S, Iwasaki Y, Ohba K, Toi M and Yamamoto N: Stromal cell-derived factor-1 and CXCR4 receptor interaction in tumor growth and metastasis of breast cancer. Biomed Pharmacother 60: 273-276, 2006.

36. Yasumoto K, Koizumi K, Kawashima A, et al: Role of the CXCL12/CXCR4 axis in peritoneal carcinomatosis of gastric cancer. Cancer Res 66: 2181-2187, 2006.

37. Kucia M, Reca R, Miekus K, et al: Trafficking of normal stem cells and metastasis of cancer stem cells involve similar mechanisms: pivotal role of the SDF-1-CXCR4 axis. Stem Cells 23: 879-894, 2005.

38. El-Andaloussi S, Lee Y, Lakhal-Littleton S, et al: Exosomemediated delivery of siRNA in vitro and in vivo. Nat Protoc 7: 2112-2126, 2012

39. Hamada K, Monnai M, Kawai K, et al: Liver metastasis models of colon cancer for evaluation of drug efficacy using NOD/ Shi-scid IL2R $\gamma^{\text {null }}$ (NOG) mice. Int J Oncol 32: 153-159, 2008.

40. Voisin T, El Firar A, Fasseu M, et al: Aberrant expression of OX1 receptors for orexins in colon cancers and liver metastases: an openable gate to apoptosis. Cancer Res 71: 3341-3351, 2011.

41. Moreno A, López LA, Fabra A and Arús C: 1H MRS markers of tumour growth in intrasplenic tumours and liver metastasis induced by injection of HT-29 cells in nude mice spleen. NMR Biomed 11: 93-106, 1998.

42. Van Niel G, Raposo G, Candalh C, et al: Intestinal epithelial cells secrete exosome-like vesicles. Gastroenterology 121: 337-349, 2001.

43. Suetsugu A, Honma K, Saji S, Moriwaki H, Ochiya T and Hoffman RM: Imaging exosome transfer from breast cancer cells to stroma at metastatic sites in orthotopic nude-mouse models. Adv Drug Deliv Rev 65: 383-390, 2013.

44. Nazarenko I, Rana S, Baumann A, et al: Cell surface tetraspanin Tspan8 contributes to molecular pathways of exosome-induced endothelial cell activation. Cancer Res 70: 1668-1678, 2010.

45. Ratajczak J, Wysoczynski M, Hayek F, Janowska-Wieczorek A and Ratajczak MZ: Membrane-derived microvesicles: important and underappreciated mediators of cell-to-cell communication. Leukemia 20: 1487-1495, 2006.

46. Fidler IJ: Orthotopic implantation of human colon carcinomas into nude mice provides a valuable model for the biology and therapy of metastasis. Cancer Metastasis Rev 10: 229-243, 1991.

47. Giavazzi R, Campbell DE, Jessup JM, Cleary K and Fidler IJ: Metastatic behavior of tumor cells isolated from primary and metastatic human colorectal carcinomas implanted into different sites in nude mice. Cancer Res 46: 1928-1933, 1986.

48. Balaj L, Lessard R, Dai L, et al: Tumour microvesicles contain retrotransposon elements and amplified oncogene sequences. Nat Commun 2: 180, 2011.

49. Aliotta JM, Pereira M, Johnson KW, et al: Microvesicle entry into marrow cells mediates tissue-specific changes in mRNA by direct delivery of mRNA and induction of transcription. Exp Hematol 38: 233-245, 2010. 
50. Aliotta JM, Sanchez-Guijo FM, Dooner GJ, et al: Alteration of marrow cell gene expression, protein production, and engraftment into lung by lung-derived microvesicles: a novel mechanism for phenotype modulation. Stem Cells 25: 2245-2256, 2007.

51. Grange C, Tapparo M, Collino F, et al: Microvesicles released from human renal cancer stem cells stimulate angiogenesis and formation of lung premetastatic niche. Cancer Res 71: 5346-5356, 2011.
52. Zeelenberg IS, Ruuls-Van Stalle L and Roos E: The chemokine receptor CXCR4 is required for outgrowth of colon carcinoma micrometastases. Cancer Res 63: 3833-3839, 2003.

53. Tamamura H, Hori A, Kanzaki N, et al: T140 analogs as CXCR4 antagonists identified as anti-metastatic agents in the treatment of breast cancer. FEBS Lett 550: 79-83, 2003.

54. Bertolini F, Dell'Agnola C, Mancuso P, et al: CXCR4 neutralization, a novel therapeutic approach for non-Hodgkin's lymphoma. Cancer Res 62: 3106-3112, 2002. 